\title{
Comparación de diferentes algoritmos metaheurísticos en la estimación de parámetros del modelo relacional general de cromatografía líquida en columna
}

\author{
Comparison between different metaheuristic algorithms in parameter estimation of \\ the general relational model of column liquid chromatography
}

\author{
Reynier Hernández Torres ${ }^{1} \quad$ Mirtha Irizar Mesa $^{1} \quad$ Orestes Llanes Santiago $^{1}$ \\ Leôncio Diógenes T. Câmara ${ }^{2}$ Antônio José da Silva Neto ${ }^{2}$ \\ Lourdes M. Zumalacárregui de Cárdenas ${ }^{3}$
}

Recibido 4 de julio de 2012, aceptado 13 de junio de 2013

Received: July 4, 2012 Accepted: June 13, 2013

\begin{abstract}
RESUMEN
La optimización, el escalamiento y la estimación de parámetros son problemas inversos que aparecen en muchos procesos biotecnológicos. En la solución de los problemas inversos se han utilizado diferentes técnicas, entre ellas los algoritmos metaheurísticos. Estos algoritmos buscan y encuentran de manera eficiente buenas soluciones a un determinado problema, con un costo computacional razonable.

En este trabajo se aplican cuatro algoritmos metaheurísticos bien conocidos (Algoritmo Genético, Evolución Diferencial, Optimización por Colonia de Hormigas y Optimización por Enjambre de Partículas) al problema de estimar parámetros en el proceso de cromatografía líquida en columna. Se analiza la efectividad de cada método, realizando una comparación basada en diferentes criterios estadísticos. El procedimiento utilizado permite obtener valores cercanos a los parámetros reales, con un buen ajuste de las curvas generadas por el modelo a los datos experimentales. El algoritmo que obtiene mejores resultados es Evolución Diferencial.
\end{abstract}

Palabras clave: Cromatografía líquida, modelo relacional general, algoritmos metaheurísticos, problemas inversos, estimación de parámetros.

\begin{abstract}
Optimization, scaling and parameter estimation are inverse problems that appear in many biotechnological processes. In the solution of inverse problems, different techniques such as metaheuristics algorithms, have been used. These algorithms efficiently search for and find good solutions to a problem with a reasonable computational cost.

In this pape, four well-known metaheuristic algorithms (Genetic Algorithm, Differential Evolution, Ant Colony Optimization and Particle Swarm Optimization) are applied to the problem of parameter estimation in the liquid chromatography separation process. The effectiveness of each method is analyzed and performance comparison based on different statistical criteria is made. The procedure allows to obtain estimated values close to the real parameters, with a good fit of the curves generated by the model to syntetically generated experimental data. The algorithm that performs better is Differential Evolution.
\end{abstract}

Keywords: Liquid chromatography, general relational model, metaheuristic algorithms, inverse problems, parameter estimation.

1 Departamento de Automática y Computación. Facultad de Ingeniería Eléctrica. Instituto Superior Politécnico José Antonio Echeverría (CUJAE); Calle 114. № 11901. e/ Ciclovía y Rotonda. Marianao, La Habana, Cuba. CP 19390.

E-mail: reynier@electrica.cujae.edu.cu; mirtha@electrica.cujae.edu.cu; orestes@electrica.cujae.edu.cu

2 Departamento de Engenharia Mecânica e Energia. Instituto Politécnico da Universidade do Estado do Rio de Janeiro (UERJ). Nova Friburgo, Rio de Janeiro, Brasil. E-mail: dcamara@iprj.uerj.br; ajsneto@iprj.uerj.br

3 Centro de Estudios de Ingeniería de Procesos. Facultad de Ingeniería Química. Instituto Superior Politécnico José Antonio Echeverría (CUJAE).Cuba. E-mail: lourdes@quimica.cujae.edu.cu 


\section{INTRODUCCIÓN}

Para comprender los mecanismos y las leyes que rigen muchos sistemas reales se han elaborado modelos que intentan reproducir su comportamiento. Para lograr la precisión de estos modelos se requiere de parámetros que caractericen correctamente al sistema. Muchas veces, no es posible medir estos parámetros en forma experimental, o resulta muy costoso debido a que demanda tiempo y gastos de reactivos. Es necesario entonces desarrollar técnicas que permitan la obtención de estos parámetros mediante el uso de métodos computacionales, permitiendo la reducción del esfuerzo y el costo de los experimentos en laboratorios.

La estimación de parámetros desconocidos de un modelo matemático se considera un problema inverso. Los métodos de estimación ajustan los parámetros del modelo para reproducir los resultados experimentales de la mejor manera posible. En esta tarea se minimiza una función objetivo que cuantifica el valor de la diferencia entre los datos simulados por el modelo y los datos de las mediciones disponibles [1]. Estos problemas son frecuentes en aplicaciones industriales, físicas, químicas y farmacéuticas.

Para realizar la estimación de parámetros de diferentes modelos se utilizan usualmente algoritmos de optimización local y global bien conocidos (gradiente conjugado, Gauss-Newton, Levenberg-Marquardt, entre otros). Los algoritmos de optimización local realizan una búsqueda alrededor de un punto inicial, y la solución que proveen con frecuencia se encuentra alejada de la mejor solución global. Por otra parte, muchos de los algoritmos de optimización global clásicos dependen de la información del gradiente, lo que en algunos problemas no está disponible.

La estimación de parámetros en procesos biotecnológicos es un problema complicado, debido a que los modelos presentan diferentes complejidades, entre las que se encuentra la no linealidad. Estos problemas comúnmente son multimodales (no convexos) y pueden presentar grandes regiones relativamente planas. Esto pudiera hacer que los algoritmos clásicos fallen en su labor de optimización, al converger a una solución local, cuando existe una mejor solución a una distancia determinada [1-2]. También pudieran arrojar resultados satisfactorios, pero con un costo computacional muy elevado. Por lo tanto, es necesario utilizar métodos de optimización global que logren una búsqueda eficiente del óptimo global. Los algoritmos metaheurísticos son técnicas que pueden solucionar estos problemas.

La cromatografía es una ciencia que estudia la separación de diferentes sustancias, basada en las diferencias estructurales y en el fenómeno de la adsorción. La simulación de procesos cromatográficos puede utilizarse para la búsqueda de parámetros que no pueden ser determinados experimentalmente. Esto puede verse limitado en su aplicación práctica por el tamaño del sistema de ecuaciones diferenciales, y la complejidad de la solución numérica [3].

La cromatografía líquida es un método de separación común, muy importante en las industrias química, farmacéutica y biotecnológica. Se utiliza para separar uno o más componentes de una mezcla. Para la optimización y el escalado de equipamiento cromatográfico, es importante conocer precisamente cuándo y a qué concentración el producto deseado puede ser recolectado de la columna. Al cambiar las condiciones de operación de un sistema multicomponente y dependiente del tiempo, el comportamiento del proceso resulta difícil de predecir. Las simulaciones computacionales brindan una solución eficiente y económica para el análisis de estos sistemas [4]. Aun así, la precisión de la simulación depende del modelo escogido y de la calidad de los parámetros.

Los parámetros de transferencia de masa normalmente no se encuentran disponibles en la literatura y no son medidos con facilidad en los experimentos. Sin embargo, estos parámetros pueden ser estimados con cierta exactitud utilizando relaciones semiempíricas provenientes de la literatura. Como estos modelos no son muy sensibles a los parámetros de transferencia de masa, el estimarlos con un cierto grado de error no afecta, en gran parte, a los resultados [5]. Los parámetros del equilibrio de adsorción tienen mayor dificultad para estimarse. Usualmente se requiere de una estimación precisa porque tienen una influencia importante en el rendimiento del adsorbente frente a un adsorbato dado. Las isotermas para sistemas particulares en general no están disponibles en la literatura.

Sobre la estimación de parámetros en procesos cromatográficos se han realizado numerosos 
trabajos. En [6-9] algunos parámetros desconocidos se estimaron según correlaciones semiempíricas. En [10] se derivó una metodología para la estimación de algunos parámetros del proceso a partir de las curvas de ruptura experimentales, basándose en un modelo detallado adaptado a partir de [11]. Se determinan los parámetros desconocidos mediante tres clases de experimentos: el comportamiento de la mezcla externa, el comportamiento de la fase móvil y el comportamiento de la fase estacionaria. Luego, en una extensión a esta metodología que hiciera el mismo equipo de investigación, se agregó la determinación de nuevos parámetros con un cuarto experimento: el comportamiento de la adsorción [12]. En estos artículos se reportó que los parámetros no fueron estimados mediante correlaciones de ningún tipo, sino que se obtenían mediante un método inverso utilizando métodos computacionales, con muchas ventajas sobre los métodos convencionales de obtención de los parámetros. La aplicación de estas técnicas requiere gastos de recursos, debido a la cantidad de experimentos necesarios. En [3] se realizó la estimación de los parámetros de la isoterma de Langmuir y bi-Langmuir utilizando un método inverso con un número mucho menor de experimentos necesarios, pero con un costo computacional alto. Más recientemente, en [13] se aplicó el Algoritmo Genético para la estimación de algunos parámetros de un modelo cromatográfico. Nuestro grupo de investigación también aplicó el Algoritmo Genético [14] al modelo que se utilizará en este artículo. En estos trabajos se han reportado soluciones satisfactorias, pero con un alto costo computacional. Se hace necesario utilizar técnicas que disminuyan el tiempo computacional y logren resultados de los parámetros lo más precisos posibles, por lo que se decide utilizar otras técnicas metaheurísticas que han demostrado mejores resultados en numerosas aplicaciones.

El objetivo de este artículo es estudiar y comparar diferentes algoritmos metaheurísticos (Algoritmo Genético, Evolución Diferencial, Optimización por Enjambre de Partículas y Optimización por Colonia de Hormigas) en la tarea de estimar parámetros del modelo general relacional del proceso de cromatografía líquida en columna. Se determinará cuál de ellos provee la mejor solución con el menor costo computacional para este problema. En la primera sección se presenta el proceso de cromatografía líquida en columna, el modelo matemático que lo describe y las condiciones experimentales. En la segunda sección se declara el problema inverso a resolver: la estimación de parámetros. En la tercera sección se presentan los algoritmos metaheurísticos que se aplicarán en la solución del problema y se fundamenta su selección. En la cuarta sección se muestran la configuración de los experimentos de estimación de parámetros y los criterios que se utilizarán para evaluar el desempeño de cada algoritmo. En la siguiente sección se hace un análisis de los resultados obtenidos y finalmente, en la última sección se presentan las conclusiones finales del trabajo y el trabajo futuro a desarrollar.

\section{CROMATOGRAFÍA LÍQUIDA EN COLUMNA}

La cromatografía líquida en columna es un proceso de separación que se alcanza por la distribución selectiva de los componentes de una mezcla entre dos fases, una móvil constituida por un medio líquido que fluye a través de la columna y una fija o estacionaria que es el material de relleno de la columna. Cada componente de esta mezcla se separa de acuerdo con la afinidad de adsorción de cada material por el adsorbente.

Los modelos de cromatografía son útiles para comprender los sistemas existentes, para así optimizarlos y escalarlos. El modelo relacional general (MRG) es uno de los modelos que mejor refleja los mecanismos de transporte y adsorción del proceso [15].

\section{Modelo relacional general}

El MRG multicomponente incluye convección, dispersión, transferencia de masa entre la fase líquida y los macroporos de las partículas, difusión en los poros de la partícula y adsorción. Las ecuaciones se definen considerando que la columna contiene perlas porosas adsorbentes que son sólidas, esféricas y de radio uniforme. Se supone que el proceso es isotérmico y no hay gradiente de concentración en la dirección radial en la columna. Otra consideración es que existe equilibrio local para cada componente entre la superficie del poro y la fase líquida en los macroporos dentro de las partículas. Además, se supone que el coeficiente de transferencia de masa y el coeficiente de difusión son constantes e 
independientes de los efectos de la mezcla de los componentes [16-19].

Para modelar el proceso de cromatografía líquida en columna se utiliza el balance de masa del adsorbato o soluto en la fase líquida y el balance de masa del soluto adsorbido en la fase sólida. Estas resultan en dos ecuaciones diferenciales parciales acopladas, dependientes del tiempo. Para el componente $i=1 \ldots$, $N_{s}$, se define:

$$
\begin{aligned}
& -D_{b i} \frac{\partial^{2} C_{b i}}{\partial Z^{2}}+\frac{\partial C_{b i}}{\partial Z}+\frac{\partial C_{b i}}{\partial t} \\
& +\frac{3 k_{i}\left(1-\varepsilon_{b}\right)}{\varepsilon_{b} R_{p}}\left(C_{b i}-C_{p i, R=R_{p}}\right)=0 \\
& \left(1-\varepsilon_{p}\right) \frac{\partial C_{p i}^{*}}{\partial t}+\varepsilon_{p} \frac{\partial C_{p i}}{\partial t} \\
& -\varepsilon_{p} D_{p i} \frac{1}{R^{2}} \frac{\partial}{\partial R}\left(R^{2} \frac{\partial C_{p i}}{\partial R}\right)=0
\end{aligned}
$$

donde:

- $\quad N_{S}$ es el número de componentes

- $\quad C_{b i}$ es la concentración del componente $i$ en la fase móvil,

- $\quad C_{p i}$ es la concentración del componente $i$ en la fase líquida dentro de las partículas,

- $\quad C_{p i}^{*}$ es la concentración del componente $i$ en la fase sólida de la partícula,

- $D_{b i}$ es el coeficiente de dispersión axial del componente $i$,

- $D_{p i}$ es el coeficiente de difusión efectiva del componente $i$, sin incluir la porosidad,

- $\quad k_{i}$ es el coeficiente de transferencia de masa externa del componente $i$,

- $\varepsilon_{b}$ es la fracción volumétrica,

- $\varepsilon_{p}$ es la porosidad de la partícula,

- $Z$ es la coordenada axial,

- $v$ es la velocidad interticial,

- $R$ es la coordenada radial para la partícula,

- $R_{p}$ es el radio de la partícula.

La isoterma de Langmuir para sistemas multicomponentes se expresa como:

$$
C_{p i}^{*}=\frac{a_{i} C_{p i}}{1+\sum_{j=1}^{N s} b_{j} C_{p i}}
$$

donde

- $a_{i}=b_{i} C_{i}^{\infty}$ es una constante de la isoterma de Langmuir para el componente $i$,

- $\quad b_{j}$ es una constante de la isoterma de Langmuir para el componente $j$,

- $\quad C_{i}^{\infty}$ es la capacidad de adsorción para el componente $i$.

Con el objetivo de reducir el número de variables, se definen las siguientes variables y números adimensionales:

$$
\begin{gathered}
c_{b i}=C_{b i} / C_{0 i}, c_{p i}=C_{p i} / C_{0 i}, c_{p i}^{*}=C_{p i}^{*} / C_{0 i} \\
\tau=v t / L, r=R / R_{p}, z=Z / L \\
P e_{L i}=v L / D_{b i}, B i_{i}=K_{i} R_{p} / \varepsilon_{p} D_{p i} \\
\eta_{i}=\varepsilon_{p} D_{p i} L / R_{p}^{2} v, \xi_{i}=3 B i_{i} \eta_{i}\left(1-\varepsilon_{b}\right) / \varepsilon_{b}
\end{gathered}
$$

donde

- L es la longitud de la columna,

- $C_{0 i}$ es igual al valor máximo del perfil de alimentación, $\max \left\{C_{f i}(\tau)\right\}$.

Las ecuaciones (1) y (2) se transforman en:

$$
\begin{aligned}
& -\frac{1}{P e_{L i}} \frac{\partial^{2} c_{b i}}{\partial z^{2}}+\frac{\partial c_{b i}}{\partial z}+\frac{\partial c_{b i}}{\partial \tau} \\
& +\xi_{i}\left(c_{b i}-c_{p i, r=1}\right)=0 \\
& \left(1-\varepsilon_{p}\right) \frac{\partial c_{p i}^{*}}{\partial \tau}+\varepsilon_{p} \frac{\partial c_{p i}}{\partial \tau} \\
& -\eta_{i} \frac{1}{r^{2}} \frac{\partial}{\partial r}\left(r^{2} \frac{\partial c_{p i}}{\partial r}\right)=0 \\
& c_{p i}^{*}=\frac{a_{i} c_{p i}}{1+\sum_{j=1}^{N_{s}}\left(b_{j} C_{0 i}\right) c_{p i}}
\end{aligned}
$$

El número de Peclet $P e_{L i}$ refleja la proporción de la velocidad de convección respecto de la velocidad de dispersión, mientras que el número de Biot $B i_{i}$ refleja la proporción de la transferencia de masa externa respecto de la velocidad de difusión dentro de la partícula. Los términos $\eta_{i}$ y $\xi_{i}$ son constantes adimensionales.

La columna está inicialmente equilibrada. Las condiciones iniciales y de contorno de las ecuaciones adimensionales son: 


$$
\begin{gathered}
\tau=0, c_{b i}=c_{b i}(0, z), c_{p i}=c_{p i}(0, r, z) \\
z=0, \frac{\partial c_{b i}}{\partial z}=P e_{L i}\left(c_{b i}-\frac{C_{f i}(\tau)}{C_{0 i}}\right) \\
z=1, \frac{\partial c_{b i}}{\partial z}=0 \\
r=0, \frac{\partial c_{p i}}{\partial r}=0 \\
r=1, \frac{\partial c_{p i}}{\partial r}=B i_{i}\left(c_{b i}-c_{p i, r=1}\right)
\end{gathered}
$$

El modelo descrito no tiene solución analítica. Para solucionar numéricamente el modelo es necesario discretizar los ejes espaciales $z$ y $r$. La ecuación de la fase móvil se discretiza utilizando el método de elementos finitos mediante $N_{z}$ nodos cuadráticos. La ecuación de la fase estacionaria se discretiza según el método de colocación ortogonal mediante $N_{r}$ puntos interiores. El procedimiento de discretización para $N_{s}$ especies (componentes) alcanza un total de $N_{s} N_{z}\left(N_{r}+1\right)$ ecuaciones diferenciales ordinarias. Por último, estas ecuaciones se resuelven simultáneamente por algún solucionador de ecuaciones diferenciales ordinarias rígidas.

Al dar solución al modelo se obtiene la historia del efluente (cromatograma o curva de ruptura). La variable obtenida es la concentración a la salida del cromatógrafo $C_{b i} \mid(z=1)$ respecto del tiempo adimensional $\tau$. El instante donde $C / C_{0 i}=0,1$ se conoce como punto de ruptura. Para el análisis de la ruptura (adsorción frontal) se mantiene $C_{f i}(\tau) / C_{0 i}=1$.

\section{Condiciones experimentales}

Todos los datos para la experimentación fueron tomados de [20]. Se utilizó una columna de diámetro interno $d_{c}=0,5 \mathrm{~cm}$ por longitud $L=3 \mathrm{~cm}$, empaquetada con una resina con perlas adsorbentes de diámetro medio $d_{p}=46 \mu \mathrm{m}$. La porosidad del lecho es $\varepsilon_{b}=0,53$ y la porosidad de la partícula $\varepsilon_{p}=0,63$.

Se utilizó un solo componente en la muestra inyectada ( $\alpha$-lactoalbúmina). El valor de concentración inicial es $C_{0}=3,5 \mathrm{mg} / \mathrm{mL}$ y el flujo volumétrico de alimentación es $Q=0,5 \mathrm{~mL} / \mathrm{min}$. Los valores reales de los parámetros para el componente se muestran en la Tabla 1. Se elimina el subíndice $i$ de la notación de los parámetros, por ser $N_{s}=1$.
Los datos de la curva de ruptura experimental fueron generados in silico (creados en la computadora mediante la solución directa del modelo).

Tabla 1. Valores reales de los parámetros.

\begin{tabular}{|c|c|c|c|c|c|}
\cline { 2 - 6 } \multicolumn{1}{c|}{} & $P e_{L}$ & $B i$ & $\eta$ & $a$ & $b$ \\
\hline Real & 247,66 & 10,07 & 1,150 & 66,19 & 0,926 \\
\hline
\end{tabular}

\section{ESTIMACIÓN DE PARÁMETROS}

Para el proceso estudiado se propone la estimación de los parámetros que definen los mecanismos de transferencia de masa y de adsorción del modelo de cromatografía líquida en columna. Se define el vector de incógnitas $\zeta=\left\{P e_{L}, B i, \eta, a, b\right\}$.

El objetivo de la estimación de parámetros consiste en encontrar un conjunto de parámetros $\zeta$ que minimice una función objetivo basada en el error entre la salida del proceso y la salida predicha por el modelo. Inicialmente, se parte de una población de soluciones candidatas generada aleatoriamente en un rango definido por $\left(\zeta_{I}, \zeta_{U}\right)$, siendo estos el límite superior e inferior para el conjunto de parámetros, respectivamente. Los parámetros se estiman numéricamente según:

$$
\hat{\zeta}=\operatorname{argmin}[J(\zeta)]
$$

La función objetivo $J(\zeta)$ mide el ajuste de los datos experimentales $y_{m}(t)$ con los valores predichos por el modelo $\tilde{y}(t, \zeta)$. Usualmente se utiliza la suma de los errores cuadráticos definida en la ecuación (7).

$$
J(\zeta)=\sum_{j=1}^{N}\left(y_{m}(t)-\tilde{y}(t, \zeta)\right)^{2}
$$

Esta función objetivo se minimiza mediante un optimizador; en este caso se utilizarán algoritmos metaheurísticos.

\section{ALGORITMOS METAHEURÍSTICOS}

Los algoritmos metaheurísticos son métodos estocásticos de búsqueda global. Pueden localizar eficientemente la vecindad del óptimo global la mayoría de las ocasiones en un tiempo computacional aceptable. Existe un gran número de algoritmos metaheurísticos, en sus versiones originales y mejoradas. 
En este artículo se seleccionaron cuatro algoritmos metaheurísticos poblacionales (dos evolutivos: Algoritmo Genético y Evolución Diferencial, y dos de inteligencia de enjambres: Optimización por Enjambre de Partículas y Optimización por Colonia de Hormigas). Estos algoritmos son bien conocidos en el mundo de la optimización global y han sido utilizados en diferentes aplicaciones similares a la de este trabajo [21].

\section{Algoritmo genético}

El Algoritmo Genético (AG) se basa en la aplicación de los principios de la biología evolutiva en la ciencia de la computación. Utiliza métodos derivados de la herencia, la mutación, la selección natural y la recombinación [21-24]. Fue inicialmente desarrollado por John H. Holland [25], investigador de la Universidad de Michigan.

El Algoritmo 1 muestra el pseudocódigo de AG.

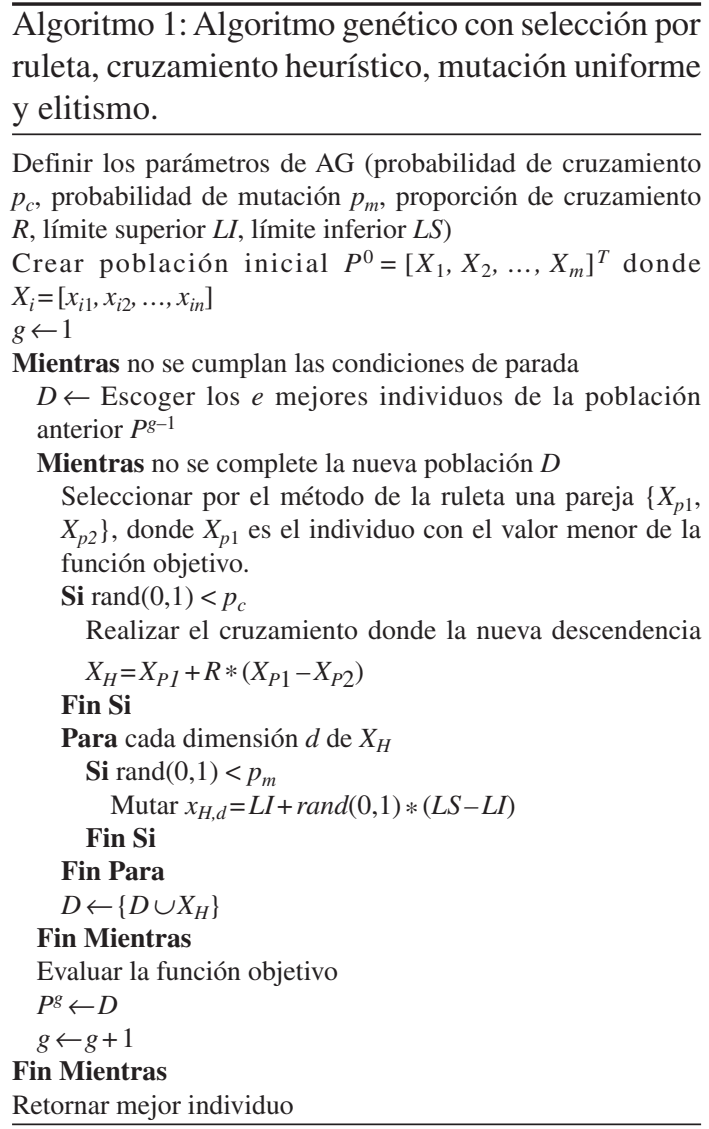
ruleta, cruzamiento heurístico, mutación uniforme y elitismo.

Definir los parámetros de AG (probabilidad de cruzamiento $p_{c}$, probabilidad de mutación $p_{m}$, proporción de cruzamiento Crear población inicial $P^{0}=\left[X_{1}, X_{2}, \ldots, X_{m}\right]^{T}$ donde $X_{i}=\left[x_{i 1}, x_{i 2}, \ldots, x_{i n}\right]$

$D \leftarrow$ Escoger los $e$ mejores individuos de la población anterior $P^{g-1}$

Mientras no se complete la nueva población $D$ unción objetivo.

i $\operatorname{rand}(0,1)<p$ $X_{H}=X_{P 1}+R *\left(X_{P 1}-X_{P 2}\right.$

Si rand $(0,1)<p_{m}$

Mutar $x_{H, d}=L I+\operatorname{rand}(0,1) *(L S-L I)$

$D \leftarrow\left\{D \cup X_{H}\right\}$

Fin Mientras

Evaluar la función objetivo

$P^{g} \leftarrow D$

Fin Mientras

Retornar mejor individuo

\section{Evolución diferencial}

Evolución Diferencial (ED), propuesto por Storn y Price [26], es un algoritmo estocástico que utiliza métodos derivados de la biología como la herencia, la mutación, la selección natural y la recombinación (o cruzamiento) para trabajar con el conjunto de soluciones. A diferencia de AG, esta técnica obtiene una nueva generación a partir de que los individuos de una población se recombinen y evolucionen [21-22, 26-27].

En este artículo se utiliza la configuración best/l/ exp, descrita a continuación.

El Algoritmo 2 muestra el pseudocódigo de ED.

$\overline{\text { Algoritmo 2: Evolución diferencial según la }}$
configuración best/l/exp

Definir los parámetros de ED (probabilidad de cruzamiento $\mathrm{Cr}$, factor de peso $F \in[0,2]$ )

Crear población inicial $P^{0}=\left[X_{1}, X_{2}, \ldots, X_{m}\right]^{T}$ donde $X_{i}=\left[x_{i 1}, x_{i 2}, \ldots, x_{i n}\right]$

Mientras no se cumplan las condiciones de parada

$X_{t} \leftarrow \varnothing$

Para cada individuo $i$ de $P^{g}$

Aplicar el operador de mutación al mejor individuo $X_{\text {mejor }}$ a partir de otros dos escogidos al azar $X_{r 1}$ y $X_{r 2}$ según la función $X_{t}=X_{\text {mejor }}+F\left(X_{r 1}+X_{r 2}\right)$

Para cada dimensión d de $X_{i} / /$ cruzamiento

Si rand $(0,1)<C r$

$x_{i d}=x_{t, d}$

Salir del Para

Sino

$x_{i d}=x_{i d}$

Fin Si

Fin Para

Evaluar la función objetivo

Si $J\left(X_{t, i}\right)<J\left(X_{i}\right) / /$ selección

$$
x_{i}=x_{t, i}
$$

Sino

$x_{i}=x_{i}$

Fin Si

Fin Para

Fin Mientras

Retornar mejor individuo

\section{Optimización por enjambre de partículas}

El algoritmo de Optimización por Enjambre de Partículas (OEP) está inspirado en el comportamiento social del vuelo de las aves en grupo o el movimiento conjunto de los peces. Esta técnica fue desarrollada por James Kennedy y Russell Eberhart en 1995 [28]. Este algoritmo mantiene un conjunto de soluciones, llamadas partículas, que son inicializadas aleatoriamente en el espacio de búsqueda y que 
comparten información entre ellas de su posición y aptitud.

Cada partícula del enjambre aprende del éxito de sus vecinos y también contribuye al mismo. Este hecho requiere de un balance entre la exploración (capacidad de búsqueda individual) y la explotación (capacidad de aprender de sus vecinos). Si hay poca exploración, el algoritmo convergerá en el primer óptimo encontrado. Por otra parte, si hay poca explotación, nunca convergerá. Las partículas deben ser individualistas y a la vez capaces de aprender de sus vecinos para así encontrar los mejores resultados [21-22, 28-29].

El Algoritmo 3 muestra el pseudocódigo de OEP.

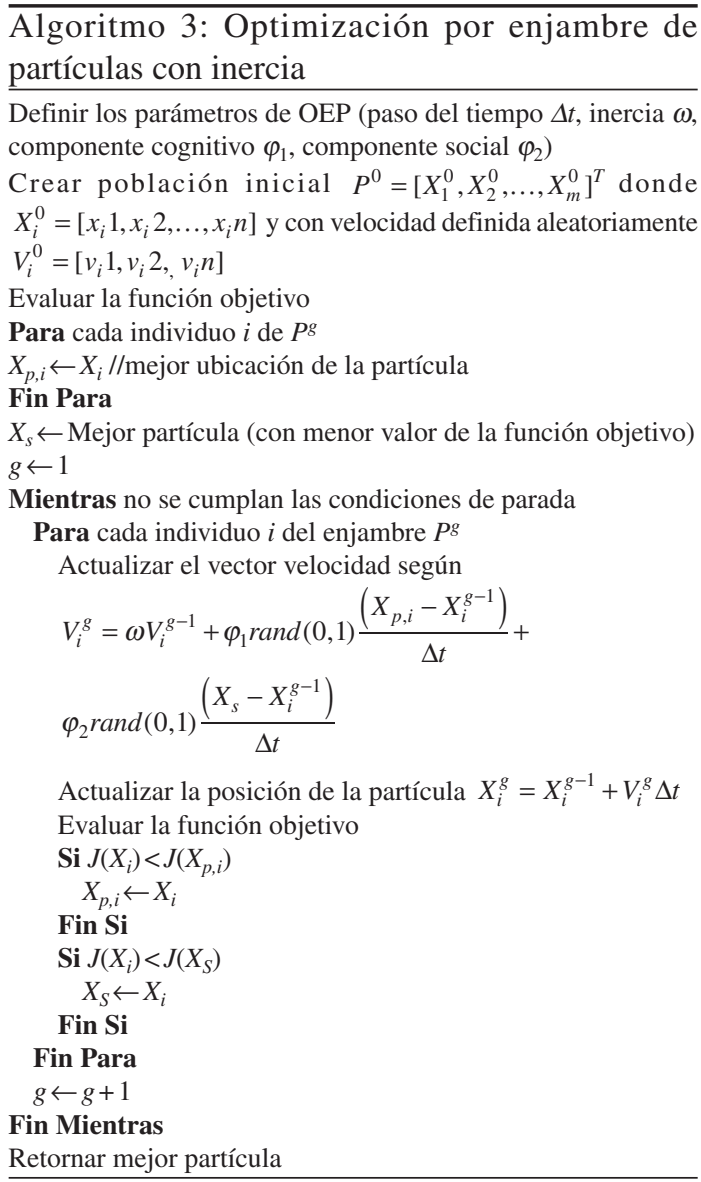

\section{Optimización por colonia de hormigas}

La Optimización por Colonias de Hormigas $(\mathrm{OCH})$ está inspirada en el comportamiento y el modo de comunicación de las hormigas. El primer algoritmo de OCH fue propuesto por Marco Dorigo en 1992
[30]. En analogía con el ejemplo biológico, este algoritmo está basado en la comunicación indirecta de una colonia de hormigas artificiales mediante los rastros de feromona artificial. Estos rastros sirven como información utilizada por las hormigas para construir soluciones candidatas para el problema [21-22, 31-32]. En los inicios todos los métodos OCH propuestos trataban con problemas combinatorios. Luego, surgió la necesidad de aplicarlo a problemas con variables continuas. El rastro de feromonas es modelado como una cantidad de feromona dejada en el camino. Esto puede ser alcanzado utilizando una función de distribución probabilística normal, como fue sugerido en [32].

El Algoritmo 4 muestra el pseudocódigo de $\mathrm{OCH}$ para dominios continuos.

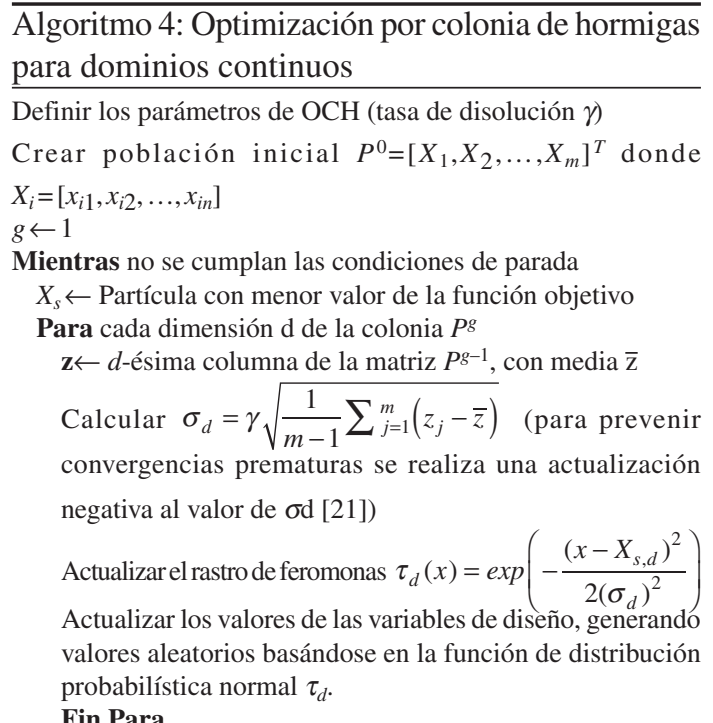
Fin Para

Evaluar la función objetivo

$g \leftarrow g+1$

Fin Mientras

Retornar mejor hormiga

\section{CONFIGURACIÓN DE LOS EXPERIMENTOS DE ESTIMACIÓN DE PARÁMETROS}

Para los experimentos de estimación de parámetros se utilizó el asistente matemático Matlab de la compañía Mathworks en su versión R2010b, corriendo sobre Microsoft Windows 7 Ultimate. Como utilidad para la optimización se utilizó el conjunto de herramientas Simple Optimization Toolbox desarrollado por [21]. Todos los cálculos fueron realizados en una computadora personal con 
procesador Intel Core 2 Duo T5500 a 1,67 GHz y 1,0 GB de memoria.

En el proceso de estimación de parámetros se realizaron corridas con poblaciones de diferentes tamaños: 20, 40, 60, 80 y 100 individuos para cada algoritmo, donde cada individuo tiene 5 dimensiones $(X=\zeta)$. La población es inicializada aleatoriamente dentro del espacio de búsqueda. Se establecieron como criterios de parada un máximo de 6000 evaluaciones de $J(\zeta)$, un límite de 20 iteraciones sin mejorar $J(\zeta)$, además de un valor a alcanzar $J_{\min }(\zeta) 1,0 \times 10^{-7}$. Los parámetros de control de cada algoritmo (tomados según la experiencia de los autores en la aplicación de estos algoritmos a la solución de este problema) se muestran en la Tabla 2.

El espacio de búsqueda se definió según se muestra en la Tabla 3. Se establecieron límites inferiores (LI) y superiores (LS) para cada parámetro, teniendo en cuenta la naturaleza física del problema.

\section{Criterios de evaluación}

Para realizar la comparación de los algoritmos se utiliza el valor promedio de la suma de los errores cuadráticos (SEC), ya definida con anterioridad según la ecuación (7).

Además, se utiliza una prueba estadística no paramétrica (la suma de los rangos de Wilcoxon)

Tabla 2. Parámetros de control de los algoritmos metaheurísticos.

\begin{tabular}{|c|c|c|}
\cline { 2 - 3 } \multicolumn{1}{c|}{} & Parámetro & Valor \\
\hline \multirow{4}{*}{ AG } & Elitismo & Dos individuos \\
\cline { 2 - 3 } & Selección & Por Ruleta \\
\cline { 2 - 3 } & $p_{c}$ & 0,8 \\
\cline { 2 - 3 } & $p_{m}$ & 0,05 \\
\cline { 2 - 3 } & $R$ & 1,2 \\
\hline $\mathbf{O C H}$ & $\gamma$ & 1,25 \\
\hline \multirow{3}{*}{ OEP } & $\omega$ & 1,4 \\
\cline { 2 - 3 } & $\varphi_{1}$ & 1,5 \\
\cline { 2 - 3 } & $\varphi_{2}$ & 2,5 \\
\hline \multirow{2}{*}{ ED } & $F$ & 0,8 \\
\cline { 2 - 3 } & $C r$ & 0,5 \\
\hline
\end{tabular}

Tabla 3. Espacio de búsqueda.

\begin{tabular}{|c|c|c|c|c|c|}
\cline { 2 - 6 } \multicolumn{1}{c|}{} & $\boldsymbol{P e}_{\boldsymbol{L}}$ & $\boldsymbol{B i}$ & $\eta$ & $\boldsymbol{a}$ & $\boldsymbol{b}$ \\
\hline $\mathbf{L I}$ & 25 & 1 & 0,11 & 6 & 0,09 \\
\hline $\mathbf{L S}$ & 470 & 20 & 2,20 & 125 & 1,75 \\
\hline
\end{tabular}

para determinar cuál de los algoritmos es el mejor. Para dos algoritmos A y B la distribución de sus resultados es comparada utilizando la hipótesis nula $H_{0}: \theta_{\mathrm{A}}=\theta_{\mathrm{B}}$ y la alternativa $H_{1}: \theta_{\mathrm{A}}<\theta_{\mathrm{B}}$, siendo $\theta$ la mediana de las observaciones de una muestra. Se realiza la prueba estadística con un nivel de significancia $\alpha=0,05$.

\section{ANÁLISIS DE LOS RESULTADOS}

La corrida de cada método de estimación de parámetros se ejecutó 25 veces. Los resultados mostrados fueron los mejores obtenidos para cada algoritmo, siendo coincidentemente los alcanzados para 60 individuos en todos los algoritmos. Es de destacar que ninguna de las corridas se detuvo por el criterio del valor a alcanzar $J_{\min }(\zeta)$ establecido, pero sí se alcanzaron valores cercanos.

Tabla 4. Resultados de la prueba de la suma de los rangos de Wilcoxon.

\begin{tabular}{|c|c|c|c|c|}
\hline & \multicolumn{3}{|c|}{ ED vs. } \\
\hline & & OCH & OEP & AG \\
\hline \multirow{4}{*}{$J(\zeta)$} & $R$ & 325 & 411 & 375 \\
\hline & $Z$ & $-6,05$ & $-4,39$ & $-5,08$ \\
\hline & $p$ & $1,42 \times 10^{-9}$ & $1,16 \times 10^{-5}$ & $3,70 \times 10^{-7}$ \\
\hline & $\mathrm{H}$ & 1 & 1 & 1 \\
\hline \multirow{4}{*}{$\% P e_{L}$} & $R$ & 537 & 669 & 627 \\
\hline & $Z$ & $-1,94$ & 0,60 & $-0,19$ \\
\hline & $p$ & 0,0523 & 0,5475 & 0,8462 \\
\hline & $\mathrm{H}$ & 0 & 0 & 0 \\
\hline \multirow{4}{*}{$\% B i$} & $R$ & 418 & 493 & 473 \\
\hline & $Z$ & $-4,25$ & $-2,79$ & $-3,18$ \\
\hline & $p$ & $2,15 \times 10^{-5}$ & 0,0052 & 0,0015 \\
\hline & $\mathrm{H}$ & 1 & 1 & 1 \\
\hline \multirow{4}{*}{$\% \eta$} & $R$ & 447 & 552 & 482 \\
\hline & $Z$ & $-3,69$ & $-1,65$ & $-3,01$ \\
\hline & $p$ & 0,0002 & 0,0991 & 0,0026 \\
\hline & $\mathrm{H}$ & 1 & 0 & 1 \\
\hline \multirow{4}{*}{$\% a$} & $R$ & 451 & 525 & 503 \\
\hline & $Z$ & $-3,61$ & $-2,17$ & $-2,60$ \\
\hline & $p$ & 0,0003 & 0,0298 & 0,0093 \\
\hline & $\mathrm{H}$ & 1 & 1 & 1 \\
\hline \multirow{4}{*}{$\% b$} & $R$ & 452 & 527 & 504 \\
\hline & $Z$ & $-3,59$ & $-2,13$ & $-2,58$ \\
\hline & $p$ & 0,0003 & 0,0328 & 0,0099 \\
\hline & $\mathrm{H}$ & 1 & 1 & 1 \\
\hline
\end{tabular}

Se realiza la prueba estadística de la suma de rangos de Wilcoxon para comparar el desempeño de los 
algoritmos y la calidad de los resultados que se obtuvieron. La Tabla 4 muestra los resultados de la prueba, donde ED es comparado contra el resto, por ser este el que presumiblemente ha obtenido los mejores resultados de convergencia. El campo $\mathrm{H}$ indica si se rechaza (valor 1) o no (valor 0) la hipótesis nula. En la primera prueba se analizan los resultados dados por el valor de $J(\zeta)$. En este caso, se obtiene que el desempeño de ED es, sin dudas, mejor que el de los otros algoritmos. En el resto de las pruebas realizadas se determina si ED obtiene parámetros con valores más cercanos a los reales, dado por el porcentaje de alejamiento del valor real. Se observa que, para el parámetro $P e_{L}$, no se tienen suficientes pruebas para rechazar la hipótesis nula. Esto ocurre también para el caso del parámetro $\eta$, cuando se compara ED con OEP. Para el resto de los casos, ED obtiene resultados mejores que los demás algoritmos.

En la Tabla 5 se muestran diferentes criterios estadísticos para comparar los resultados obtenidos por cada algoritmo. Se observa el conjunto de parámetros reales, la mejor y la peor corrida, la mediana de $J(\zeta)$ para cada algoritmo, el promedio para todas las corridas realizadas, el intervalo de confianza al 95\% (IC), la desviación estándar (STD) y el coeficiente de variación $(\mathrm{CV}=$ Promedio/STD) para cada parámetro. El valor promedio de la función objetivo corresponde al promedio de los valores de la función objetivo de cada corrida, no al valor de la corrida con el valor promedio de los parámetros. Se

Tabla 5. Estadísticas de los resultados de la estimación de parámetros. Los mejores resultados individuales para cada parámetro son marcados en negrita. La mejor corrida entre los cuatro algoritmos es marcada en negrita cursiva. El mejor algoritmo resulta ser el Evolución Diferencial.

\begin{tabular}{|c|c|c|c|c|c|c|c|c|c|c|c|c|}
\hline \multirow{2}{*}{ Algoritmo } & \multirow{2}{*}{ Estadística } & \multicolumn{10}{|c|}{ Parámetros $(\zeta)$} & \multirow{2}{*}{$J(\zeta)$} \\
\hline & & $P e_{L}$ & $\%$ e & $B i$ & $\%$ e & $\eta$ & $\%$ e & $a$ & $\%$ e & $b$ & $\%$ e & \\
\hline \multicolumn{2}{|r|}{ Real } & \multicolumn{2}{|c|}{247,66} & \multicolumn{2}{|c|}{10,07} & \multicolumn{2}{|c|}{1,15} & \multicolumn{2}{|c|}{66,19} & \multicolumn{2}{|c|}{0,926} & - \\
\hline \multirow{7}{*}{ OCH } & Mejor corrida & 179,173 & 27,7 & 8,334 & 17,2 & 1,297 & 12,8 & 62,690 & 5,3 & 0,865 & 6,6 & $3,28 \times 10^{-4}$ \\
\hline & Peor corrida & 44,950 & 81,9 & 5,281 & 47,6 & 1,531 & 33,1 & 98,818 & 49,3 & 1,530 & 65,2 & $4,22 \times 10^{-3}$ \\
\hline & Promedio & 265,389 & 7,2 & 11,393 & 13,1 & 1,253 & 9,0 & 68,061 & 2,8 & 0,959 & 3,6 & $1,31 \times 10^{-3}$ \\
\hline & IC & \multicolumn{2}{|c|}{ $\pm 55,246$} & \multicolumn{2}{|c|}{ $\pm 1,346$} & \multicolumn{2}{|c|}{ $\pm 0,123$} & \multicolumn{2}{|c|}{ $\pm 8,244$} & \multicolumn{2}{|c|}{ $\pm 0,152$} & - \\
\hline & Mediana & \multicolumn{2}{|c|}{28,1759} & \multicolumn{2}{|c|}{12,5049} & \multicolumn{2}{|c|}{1,7799} & \multicolumn{2}{|c|}{59,7148} & \multicolumn{2}{|c|}{0,8081} & $1,00 \times 10^{-3}$ \\
\hline & STD & \multicolumn{2}{|c|}{133,838} & \multicolumn{2}{|c|}{3,260} & \multicolumn{2}{|c|}{0,299} & \multicolumn{2}{|c|}{19,972} & \multicolumn{2}{|c|}{0,368} & - \\
\hline & CV (\%) & \multicolumn{2}{|c|}{50,4} & 28 & & 23, & & 29, & & 38 , & & - \\
\hline & Mejor corrida & 165,601 & 33,1 & 10,636 & 5,6 & 1,181 & 2,7 & 64,403 & 2,7 & 0,893 & 3,6 & $1,29 \times 10^{-6}$ \\
\hline & Peor corrida & 324,347 & 31,0 & 6,028 & 40,1 & 1,513 & 31,6 & 57,708 & 12,8 & 0,773 & 16,5 & $5,88 \times 10^{-4}$ \\
\hline & Promedio & 238,241 & 3,8 & 10,080 & 0,1 & 1,138 & 1,0 & 73,635 & 11,2 & 1,063 & 14,8 & $1,33 \times 10^{-4}$ \\
\hline OEP & IC & $\pm 37,2$ & & $\pm 0,7$ & & \pm 0, & 59 & $\pm 5,7$ & & $\pm 0,1$ & 106 & - \\
\hline & Mediana & 285,33 & & 10,9 & & 1,03 & & 76,17 & & 1,10 & & $8,49 \times 10^{-5}$ \\
\hline & STD & 90,13 & & 1,9 & & 0,1 & & 14,0 & & 0,2 & & - \\
\hline & $\mathrm{CV}(\%)$ & 37,8 & & 18 , & & 12 & & 19 , & & 24, & & - \\
\hline & Mejor corrida & 306,611 & 23,8 & 9,723 & 3,4 & 1,108 & 3,7 & 71,130 & 7,5 & 1,017 & 9,8 & $5,40 \times 10^{-6}$ \\
\hline & Peor corrida & 229,112 & 7,5 & 7,396 & 26,6 & 1,285 & 11,7 & 69,231 & 4,6 & 0,979 & 5,7 & $1,51 \times 10^{-3}$ \\
\hline & Promedio & 212,892 & 14,0 & 9,977 & 0,9 & 1,207 & 5,0 & 71,686 & 8,3 & 1,027 & 10,9 & $2,56 \times 10^{-4}$ \\
\hline AG & IC & $\pm 41,2$ & & \pm 0, & & $\pm 0,0$ & & $\pm 6,0$ & & $\pm 0,1$ & 112 & - \\
\hline & Mediana & 213,82 & & 8,41 & & 1,56 & & 46,98 & & 0,57 & & $1,10 \times 10^{-4}$ \\
\hline & STD & 99,97 & & 2,3 & & 0,1 & & 14,7 & & 0,27 & & - \\
\hline & $\mathrm{CV}(\%)$ & 47,0 & & 23, & & 16 & & 20 & & 26 , & & - \\
\hline & Mejor corrida & 418,088 & 68,8 & 9,488 & 5,8 & 1,148 & 0,2 & 66,278 & 0,1 & 0,928 & 0,2 & $1,02 \times 10^{-7}$ \\
\hline & Peor corrida & 141,352 & 42,9 & 9,415 & 6,5 & 1,210 & 5,2 & 67,607 & 2,1 & 0,954 & 3,0 & $8,10 \times 10^{-5}$ \\
\hline & Promedio & 252,404 & 1,9 & 9,985 & 0,8 & 1,168 & 1,6 & 67,539 & 2,0 & 0,951 & 2,7 & $1,83 \times 10^{-5}$ \\
\hline ED & IC & $\pm 38,5$ & & $\pm 0, ?$ & & $\pm 0,0$ & 42 & $\pm 3,4$ & & $\pm 0,0$ & 63 & - \\
\hline & Mediana & 300,35 & & 10,1 & & 1,16 & & 63,89 & & 0,88 & & $4,70 \times 10^{-6}$ \\
\hline & STD & 93,45 & & 0,8 & & 0,1 & & 8,29 & & 0,15 & & - \\
\hline & $\mathrm{CV}(\%)$ & 37,0 & & 8, & & 8, & & 12 , & & 16 , & & - \\
\hline
\end{tabular}


muestra el porcentaje de error absoluto (\%e) de cada parámetro respecto del valor real. Los resultados promedios más cercanos al valor real y los menores valores de CV son señalados en negrita. El algoritmo ED alcanzó los mejores resultados en la solución de este problema. El promedio de los parámetros obtenidos para ella son los más cercanos al real, con un IC pequeño con relación al resto de los algoritmos. Además, el grado de dispersión de los resultados son los menores, dado por SEM, STD y $\mathrm{CV}$. Igualmente, este algoritmo arrojó los mejores resultados del ajuste del modelo, dados por tener la función objetivo SEC los menores valores obtenidos.

Es de destacar que, para todas las estrategias, el parámetro $P e_{L}$ presenta un alto valor de dispersión en sus estimaciones. El valor $\mathrm{CV}$ para este parámetro se encuentra por encima de $37 \%$, indicando que los valores obtenidos se encuentran en una gama bastante amplia. Este es un tema en el que se necesita profundizar en trabajos futuros.

La calidad de la convergencia en las soluciones alcanzadas se muestra en la Tabla 6. Los indicadores son el tiempo promedio de las corridas (TC), el número promedio de evaluaciones de la función objetivo (NEF) y el porcentaje de veces que un algoritmo alcanzó 10,100 y 1000 veces el valor de $J_{\min }(\zeta)(\% \mathrm{~S} 1, \% \mathrm{~S} 2$ y $\% \mathrm{~S} 3)$, respectivamente [33]. Respecto de estos indicadores, el algoritmo ED resultó ser el mejor. Se puede observar que todos los algoritmos paran antes de cumplirse el número de iteraciones máximas (NEF es menor

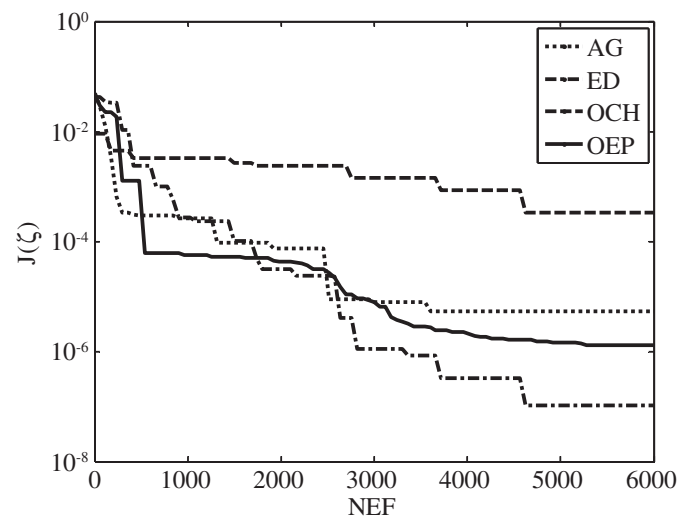

Figura 1. Gráfico de convergencia para la mejor corrida para cada algoritmo. Se muestra el valor de $J(\zeta)$ en función del número de evaluaciones de la función objetivo. que 6000 en la mayoría de las corridas). En la Figura 1 se muestra el gráfico de convergencia para la mejor corrida de cada algoritmo. En este gráfico se puede observar como el ED se mantiene mejorando el valor mínimo de la función objetivo en cada iteración con una velocidad mayor que el resto de los algoritmos. Es de destacar que en un inicio el OEP disminuye rápidamente el valor de $J(\zeta)$, pero después comienza a minimizar de una manera más suave.

El tiempo de una corrida en este problema se ve influenciado principalmente por el tiempo de ejecución del modelo del proceso, que puede variar entre 3 y 6,5 segundos cada vez y no por la ejecución del algoritmo en sí, que dura mucho menos que estas cantidades. Esto implica que a un número mayor de llamadas a la función objetivo aumenta considerablemente el tiempo de corrida, independientemente del algoritmo.

Tabla 6. Resultados de los indicadores de convergencia para los algoritmos.

\begin{tabular}{|c|c|c|c|c|c|}
\cline { 2 - 6 } \multicolumn{1}{c|}{} & TC & NEF & \%S1 & \%S2 & \%S3 \\
\hline AG & 28605,0 s (7,9 h) & 5256 & 0,0 & 4,0 & 48,0 \\
\hline ED & $\mathbf{2 6 9 2 9 , 8}$ s (7,5 h) & $\mathbf{5 0 2 1}$ & $\mathbf{8 , 0}$ & $\mathbf{6 4 , 0}$ & $\mathbf{1 0 0 , 0}$ \\
\hline OCH & 27967,3 s $(7,8$ h) & 5194 & 0,0 & 0,0 & 0,0 \\
\hline OEP & 32308,3 s $(9,0$ h $)$ & 5429 & 0,0 & 16,0 & 52,0 \\
\hline
\end{tabular}

\section{CONCLUSIONES}

La estimación de parámetros de procesos biotecnológicos es compleja. Usualmente, los modelos matemáticos que los caracterizan no presentan solución analítica. Los algoritmos de optimización clásicos fallan en la solución de este problema, llegando a soluciones locales lejos del óptimo global. Se aplican entonces algoritmos de optimización global que lidien con este tipo de complicaciones.

En este artículo se analizó la aplicación de cuatro algoritmos de optimización global (AG, ED, OCH y OEP) en la estimación de parámetros del proceso de cromatografía líquida en columna y se comparó su desempeño en la solución de este problema. Se trabajó sobre un sistema de un solo componente, utilizando datos experimentales in silico.

Estos métodos demostraron obtener valores de los parámetros del modelo con una determinada 
exactitud, mediante la computación, reduciendo las pruebas experimentales en el laboratorio. Esto trae consigo obtener resultados con un gasto reducido en recursos materiales.

En el estudio, el método basado en ED resultó ser mejor que el resto, al obtener el promedio más cercano a los valores reales y el menor grado de dispersión (dado por CV) de los parámetros estimados. Además, ED obtuvo los mejores resultados en cuanto a indicadores de convergencia.

En estudios futuros se utilizarán sistemas con dos componentes o más, para analizar el comportamiento de los algoritmos evolutivos en la estimación de parámetros del modelo del proceso de cromatografía líquida en columna bajo estas condiciones. Además, se considerarán técnicas basadas en métodos híbridos, para intentar lograr una convergencia más rápida y precisa.

Este procedimiento es aplicable a la estimación de parámetros de modelos de otros problemas complejos que no presenten una solución analítica.

\section{AGRADECIMIENTOS}

Los autores agradecen a Coordenação de Aperfeiçoamento de Pessoal de Nível Superior do Brazil (CAPES) y al Ministerio de Educación Superior de Cuba (MES). Además, se agradece la ayuda del Dr. C. T. Miladys Limonta Fernández, del Centro de Ingeniería Genética y Biotecnología (CIGB) de Cuba y a Dr. C. T. Felipe A. Chegury Viana, de la Universidade Federal de Uberlândia por facilitar el paquete Simple Optimization Toolbox 4,0.

\section{REFERENCIAS}

[1] S.M. Baker, K. Schallau and B.H. Junker. "Comparison of different algorithms for simultaneous estimation of multiple parameters in kinetic metabolic models". Journal of Integrative Bioinformatics. Vol. 7, Issue 3, pp. 1-9. 2010. DOI: 10,2390/ biecoll-jib-2010-133.

[2] M. Rodríguez-Fernández, A.A. Alonso and J.R. Banga. "Robust parameter estimation in nonlinear dynamic process models". European Symposium on Computer Aided Process Engineering. 2005.
[3] P. Forssén, R. Arnell and T. Fornstedt. "An improved algorithm for solving inverse problems in liquid chromatography". Computers and Chemical Engineering. No 30, pp. 1381-1391. 2006.

[4] P. Persson, P.-E. Gustavsson, G. Zacchi and B. Nilsson. "Aspects of estimating parameter dependencies in a detailed chromatography model based on frontal experiments". Process Biochemistry. Vol. 41, pp. 1812-1821. 2006.

[5] C. Lazo. "Simulation of Liquid Chromatography and Simulated Moving Bed (SMB) Systems". Studienarbeit, Technische Universität, Hamburg-Harburg. 1999.

[6] U. Altenhöner, M. Meurer, J. Strube and H. Schmidt-Traub. "Parameter estimation for the simulation of liquid chromatography". Journal of Chromatography A. Vol. 769, pp. 59-69. 1997.

[7] A.K. Avci, M.C. Camurdan and K.O. Ulgen. "Quantitative description of protein adsorption by frontal analysis". Process Biochemistry. Vol. 36, Issue 1-2, pp. 141-148. 2000.

[8] J.C. Bosma and J.A. Wesselingh. "Partitioning and diffusion of large molecules in fibrous structures". Journal of Chromatography B. Vol. 743, pp. 169-180. 2000.

[9] H.K. Teoh, M. Turner, N. Titchener-Hooker and E. Sorensen. "Experimental verification and optimisation of a detailed dynamic high performance liquid chromatography column model". Computers and Chemical Engineering. Vol. 25, pp. 893-903. 2001.

[10] P. Persson, H. Kempe, G. Zacchi and B. Nilsson. "A methodology for estimation of mass transfer parameters in a detailed chromatography model based on frontal experiments". Chemical Engineering Research and Design. Vol. 82, Issue A4, pp. 517-526. 2004.

[11] Z. Li, Y. Gu and T. Gu. "Mathematical modeling and scale-up of size-exclusion chromatography". Biochemical Engineering Journal. Vol. 2, pp. 145-155. 1998.

[12] P. Persson, H. Kempe, G. Zacchi and B. Nilsson. "Estimation of adsorption parameters in a detailed affinity chromatography model based on shallow bed experiments". Process Biochemistry. Vol. 40, Issue 5, pp. 1649-1659. 2005. 
[13] G. Sandoval, C. Shene, B.A. Andrews and J.A. Asenjo. "Extension of the selection of protein chromatography and the rate model to affinity chromatography". Journal of Molecular Recognition. Vol. 23, pp. 609617. 2010.

[14] R. Hernández Torres, M. Irizar Mesa, L.D.T. Câmara, A.J. da Silva Neto and O. Llanes Santiago. "Application of genetic algorithms for parameter estimation in liquid chromatography". Revista de Ingeniería Electrónica, Automática y Comunicaciones. Vol. XXXII No 3, pp. 13-20. 2011.

[15] M.E. Lienqueo, A. Mahn, J.C. Salgado and C. Shene. "Mathematical Modeling of Protein Chromatograms". Chemical Engineering Technology. Vol. 35, Issue 1, pp. 46-57. 2012.

[16] T. Gu and Y. Zheng. "A study of the scale-up of reversed-phase liquid chromatography". Separation and Purification Technology. Vol. 15, pp. 41-58. 1999.

[17] G. Guiochon. "Preparative liquid chromatography: Review". Journal of Chromatography A. Vol. 965, pp. 129-161. 2002.

[18] S. Engell and A. Toumi. "Optimization and control of chromatography". Computers and Chemical Engineering. Vol. 29, pp. 12431252. 2005.

[19] T. Gu, M. Liu, K.-S.C. Cheng, S. Ramaswamy and C. Wang. "A general rate model approach for the optimization of the core radius fraction for multicomponent isocratic elution in preparative nonlinear liquid chromatography using cored beads". Chemical Engineering Science. Vol. 66, pp. 3531-3539. 2011.

[20] S. Henriques Saraiva. "Modelagem e Simulação de Processos de Cromatografia Preparativa". Tesis de doctorado. Universidade Federal de Viçosa. 2003.

[21] F.A. Chegury Viana. "Surrogate Modeling Techniques and Heuristic Optimization Methods Applied to Design and Identification Problems". Tesis de doctorado. Universidade Federal de Uberlândia. 2008.

[22] A.J. da Silva Neto and J.C. Becceneri. "Técnicas de Inteligência Computacional Inspiradas na Natureza: Aplicações em
Problemas Inversos em Transferência Radiativa (Notas em Matemática Aplicada)". Vol. 41. Sociedade Brasileira de Matemática Aplicada e Computacional. 2009.

[23] J. M. Herrero, X. Blasco, M. Martnez and J. Sanchis. "Identification of continuous processes parameters using genetic algorithms". Proceedings of the 10th Mediterranean Conference on Control and Automation, (Lisboa, Portugal). Julio 2002.

[24] R.L. Haupt and S.E. Haupt. "Practical genetic algorithms". John Wiley \& Sons, Inc., 2nd ed. 2004.

[25] J.H. Holland. "Adaptation in Natural and Artificial Systems". Ann Arbor, MI: University of Michigan Press. 1975.

[26] R.M. Storn and K.V. Price. "Differential evolution: a simple and efficient adaptive scheme for global optimization over continuous spaces". Journal of Global Optimization. Vol. 12, pp. 1-16. 1997.

[27] K.V. Price, R.M. Storn and J.A. Lampinen. "Differential Evolution-A Practical Approach to Global Optimization". Springer. 2005.

[28] J. Kennedy and R. Eberhart. "Particle swarm optimization". IEEE International Conference on Neural Networks. Vol. 4, pp. 1942-1948. Perth, Australia. 1995.

[29] K. Kameyama. "Particle swarm optimization a survey". IEICE Transactions on Information and Systems. Vol. E92-D, Issue 7, pp. 13541361. 2009.

[30] M. Dorigo. "Optimización, aprendizaje automático y algoritmos basados en metáfora natural" (en Italiano). Tesis de doctorado, Politecnico di Milano. Milano, Italia. 1992.

[31] C. Blum. "Ant colony optimization: Introduction and recent trends-Review". Physics of Life Reviews. Vol. 2, pp. 353-373. 2005.

[32] K. Socha and M. Dorigo. "ACO for Continuous and Mixed-Variable Optimization". Lecture Notes in Computer Science. Vol. 3172, pp. 25-36. 2004.

[33] N. Hansen, A. Auger, S. Finck and R. Ros, "Real-Parameter Black-Box Optimization Benchmarking 2009: Experimental Setup". Research Report. INRIA. 2009. 